\title{
An introduction to the problem of bridging quantum and classical dynamics
}

\author{
S. Bonella ${ }^{1, a}$ and G. Ciccotti ${ }^{2,3, b}$
}

1 CECAM Centre Européen de Calcul Atomique et Moléculaire, École Polytechnique Fédérale de Lausanne, Batochime, Avenue Forel 2, 1015 Lausanne, Suisse

2 Dipartimento di Fisica, Università di Roma "La Sapienza", Ple A. Moro 2, 00185 Roma, Italia

3 School of Physics, University College of Dublin UCD, Belfield, Dublin 4, Ireland

Received 26 March 2015 / Received in final form 5 May 2015

Published online 22 June 2015

\begin{abstract}
Simulating the exact quantum dynamics of realistic interacting systems is presently a task beyond reach but for the smallest of them, as the numerical cost for solving the time-dependent Schrödinger equation scales exponentially with the number of degrees of freedom. Mixed quantum-classical methods attempt to solve this problem by starting from a full quantum description of the system and subsequently partitioning the degrees of freedom in two subsets: the quantum subsystem and the bath. A classical limit is then taken for the bath while preserving, at least approximately, the quantum evolution of the subsystem. A key, as yet not fully resolved, theoretical question is how to do so by constructing a consistent description of the overall dynamics. An exhaustive review of this class of methods is beyond the scope of this paper and we shall limit ourselves to present, as an example, a specific approach, known as the LANDM-Map method. The method stems from an attempt at taking a rigorous limit for the classical degrees of freedom starting from a path integral formulation of the full quantum problem. The results that we discuss are not new, but our intent here is to present them as an introduction to the problem of mixed quantum classical dynamics. We shall also indicate a broad classification of the available approaches, their limitations, and some open questions in this field.
\end{abstract}

\section{Introduction}

Computer simulation of quantum time dependent properties. e.g. time correlation functions, is considerably less powerful than its classical counterpart. The crucial difficulty hindering it is that all available exact methods for solving the quantum time evolution equations (such as the time dependent Schrödinger equation for the wave function or, for example, the quantum Liouville equation for the density) scale

\footnotetext{
a e-mail: sara.bonella@epfl.ch

b e-mail: giovanni.ciccotti@roma1.infn.it
} 
exponentially with the number of degrees of freedom. This scaling effectively limits exact calculations to a few tenths of interacting degrees of freedom and to times of the order of the picosecond. This is to be contrasted with the millions of degrees of freedom and tenths of microseconds accessible in the classical case when an empirical description of the interactions is adopted. Overcoming these limitations is a very interesting theoretical problem. As technologies move to smaller scales or seek to exploit quantum effects, for example coherence to engineer quantum computers, it is also rapidly becoming a problem with potentially relevant applications.

Several approximate methods have been developed to increase performance in the modelling of quantum dynamics, many of which attempt to import, with appropriate modifications, classical molecular dynamics algorithms to this area. In this paper, we will focus in particular on the so-called mixed quantum classical approaches. These approaches are useful when the degrees of freedom of the system can be partitioned, for example based on the magnitude of their De Broglie wavelength, in two subsets. The first subset, often indicated as the classical bath, is characterised by a small De Broglie wavelength and can include a large number of degrees of freedom. The second, the quantum subsystem, is characterised by a large wavelength and is small enough that the quantum problem can be solved numerically. A typical situation in which this partition can be used is to describe the dynamics of systems of nuclei (the classical bath) and electrons (the quantum subsystem) beyond the Born-Oppenheimer approximation, i.e. non adiabatic systems. In these systems, the coupling between nuclear and electronic motions in a molecule, or interactions with the environment can induce transitions among the different states of the electronic subsystem. These transitions can change, for example, the products of a chemical reaction by opening reaction channels in the excited states that follow different relaxation paths depending on the set of electronic transitions that are activated. Typical situations in which they occur are reactive scattering on surfaces, environmentally controlled photochemistry and coherent population transfer in photosynthetic centres.

The general idea of mixed quantum classical methods is to start from a fully quantum representation of the overall system and take a classical limit for the dynamics of the bath, while maintaining a quantum description of the evolution of the smaller subset. The existing approaches differ essentially in three aspects: the starting quantum formalism (e.g. wave function or density matrix based, Heisenberg or Schrödinger representation...); the way in which the classical limit for the bath is taken; and, crucially, the way in which the coupling between the evolutions of the two subsets is determined. The last two aspects are particularly relevant since they are the key steps to bridge the quantum and classical scales of the problem. Two main approaches have been pursued so far: (1) assign the evolution equations (in particular the form of the force to which the nuclei are subject) and create an ad hoc coupling mechanism designed to mimic a specific physical process; (2) rigorously determine the evolution and the coupling via the procedure used to enforce the classical limit for the dynamics of the bath. Ad hoc methods have the advantage of converging ${ }^{1}$ with relatively little numerical effort, but it is very difficult, if not impossible, to assess their general reliability. Possibly, the oldest scheme of this type is Ehrenfest dynamics $[1,2]$. In this approach, the nuclei are assumed to follow classical dynamics. The force that

\footnotetext{
1 The concept of convergence is used with two meanings in this paper. (1) When exact, numerical or analytical, quantum results are available for comparison, convergence indicates that quantum-classical results are indistinguishable, within the error, from the exact result. (2) In the absence of reference results, convergence means that increasing the size of the sample does not change, within a predefined accuracy threshold, the value of the estimator of a given physical quantity. In mixed quantum classical calculations, the size of the sample is equal to the number of propagated nuclear trajectories. In case (2) convergence does not ensure accuracy, but it gives the best estimate for a given method.
} 
they experience is given by the quantum expectation value over the electronic degrees of freedom of the gradient of the potential with respect to the nuclear positions. The electrons, on the other hand, evolve with an Hamiltonian that depends parametrically on the (time dependent) nuclear positions. Such a self-consistent, mean field like, approach is well suited to describe phenomena in which the electronic energies (which depend parametrically on the nuclear positions like in standard Born-Oppenheimer) have similar gradients with respect to the nuclear coordinates. They can, however, fail quite dramatically when this is not the case. To circumvent this problem, a different method was introduced in the 1970s by John Tully: Surface Hopping [3-5]. Surface Hopping is developed in the so-called adiabatic representation ${ }^{2}$. In this representation, the electronic energies are the Born-Oppenheimer surfaces for the system, parametrically dependent on the nuclear coordinates. The algorithm aims at reproducing the evolution of the state of the system, $|\Psi(t)\rangle$, and, more specifically, at mimicking the amplitude transfer that occurs among the electronic states in the presence of non-adiabatic phenomena. This is achieved by propagating an ensemble (a swarm) of $N_{T}$ classical nuclear trajectories. The propagation of these trajectories is determined by an ansatz that combines a fully classical part, in which each trajectory moves according to Newton's equations on a single (in contrast to the mean field Ehrenfest approach) electronic surface, and a stochastic term. The stochastic term mimics quantum non-adiabatic transitions by introducing a probabilistic mechanism for the trajectory to instantaneously "hop" on another electronic surface when the coupling among states becomes significant. This mechanism attempts to provide the correct time dependent probability to find the system at a given nuclear configuration and on a specified electronic state, $p_{\alpha}(R, t)=\left|\left\langle\phi_{\alpha}(R) R \mid \Psi(t)\right\rangle\right|^{2}$, by tuning the hopping probability so that $p_{\alpha}(R, t) \approx N_{\alpha}(R, t) / N_{T}$ where $N_{\alpha}(R, t)$ is the number of trajectories in the ensemble on state $\alpha$ and at position $R$ at time $t$. (The electronic problem is solved, similar to the Ehrenfest approach, by integrating the quantum evolution of the adiabatic states as governed by an Hamiltonian, $h_{e l}$ in note 2 , which depends parametrically on the nuclear trajectory.) The hopping probability adopted in Surface Hopping is constructed based on physical arguments, but it has so far been impossible to derive it, and, importantly, the feedback mechanism between nuclear and electronic evolutions at the time of a hop, using rigorous arguments. This is at the origin of several well known pathologies of the approach which, for example, often over estimates transition probabilities due to a wrong treatment of the electronic quantum coherence. In spite of these shortcomings, Surface Hopping is still the most popular mixed quantum classical method due to its conceptual simplicity and ease of numerical implementation. Furthermore, Surface Hopping calculations converge, or at least provide meaningful information, with a relatively small number of trajectories making it possible to combine them with first principle calculations (usually based on time dependent density functional theory [6-8]) of the electronic states and couplings. Because of this, and of the fact that Surface Hopping results are often surprisingly accurate when compared to experiments, considerable efforts are devoted to improve its formal standings by deriving it as the limiting case of more rigorous methods $[9,10]$ or, perhaps more dangerously, by attempting to find a posteriori patches to mitigate its known deficiencies, in particular the coherence problem [11-13]. These efforts have indeed improved understanding of the limitations and range of applicability of the

\footnotetext{
${ }^{2}$ Let us define the Hamiltonian of the system as the sum of the nuclear kinetic energy and of an electronic Hamiltonian, which contains the electron's kinetic energy and all the interaction potentials, thus $\hat{H}=\hat{P}^{2} / 2 M+\hat{h}_{e l}(\hat{p}, \hat{r}, \hat{R})$ (capital letters indicate nuclear operators, lower case letters are for electronic operators). The adiabatic basis is defined as $|R\rangle\left|\phi_{\alpha}(R)\right\rangle$ where $\left|\phi_{\alpha}(R)\right\rangle$ are the eigenstates of the electronic Hamiltonian. The corresponding eigenvalues, $E_{\alpha}(R)$, are known as the electronic energies.
} 
method, but the ultimate goal of a rigorous derivation of Surface Hopping has not been achieved yet and remains an open question in the field.

As mentioned above, the second family of mixed quantum classical methods follows a different approach in which algorithms are derived via formal limits of the exact quantum dynamics. A typical example is the quantum-classical Liouville method [14], whose starting point is the time evolution equation for the density matrix of the full system. A phase space representation for the bath's degrees of freedom is then introduced via a partial Wigner transform in these degrees of freedom ${ }^{3}$, while retaining a fully abstract form for the subsystem. The quantum evolution operator in this mixed representation is then expanded to first order in the parameter $\mu=\sqrt{m / M}(m$ and $M$ are the masses of the subsystem and the bath, respectively) to obtain the generator of the mixed quantum classical dynamics. This generator is given by a generalised Lie bracket in which both a commutator (acting on the subsystem operators) and a Poisson parenthesis (acting on the bath's variables) appear. Once a basis set is chosen for the subsystem (for example the adiabatic basis mentioned above) the evolution equation for the density matrix, or for any other operator, becomes explicit and different algorithms have been proposed to solve it $[16,17]$. These algorithms share the characteristic that the bath motion is obtained via a classical evolution, possibly including generalised definitions of the force that describe the influence of more than one electronic state on the trajectory. The quantum-classical Liouville evolution does not suffer from some of the limitations of Surface Hopping. For example, it can provide both diagonal and off-diagonal elements of the density matrix of the system. The latter are related to the electronic coherence which is then naturally included, within the limits of the approximation, in the description of the system. In spite of its merits, however, it has been shown that this mixed quantum classical dynamics lacks several properties that characterise fully classical and quantum dynamics [18]. In particular, the mixed Lie bracket does not satisfy the Jacobi identity exactly and does not preserve stationarity of the quantum thermal density. The loss of formal properties with respect to the purely classical and quantum cases arises, in different forms, in all current mixed schemes. Determining whether this is to be interpreted as a failure for mixed schemes or if there is no reason or need to expect or require such properties from these approximate methods is an interesting open question. Another important problem for formally derived mixed approaches is that the corresponding algorithms are still considerably, sometimes prohibitively, more expensive than classical calculations. They are also considerably more expensive than ad hoc methods, often requiring one or two orders of magnitude more trajectories to converge. While this may be considered a reasonable price to pay for the increased reliability, it is a serious obstacle to modelling multidimensional systems and prevents a wide use of these methods.

To illustrate a little more in detail formally derived mixed approaches, we shall devote the next two sections to describe another one of them. This method, known as LAND-Map or linearised density matrix approach [19], was originally developed to compute approximate time correlation functions but it can be easily adapted to density matrix or time dependent averages calculations. It combines the path integral representation of the time propagator introduced by Feynman with an appropriate representation of the electronic states as the starting point for an approximation that determines, like in mixed Liouville dynamics, both the force governing the, classical like, evolution of the nuclei and the coupling between the classical and quantum degrees of freedom. This approximation is again based on a well defined limiting procedure that will be presented below. This specific method was chosen because of the

\footnotetext{
3 The Wigner transform [15] of a quantum operator $\hat{O}$ is defined as $O_{w}(R, P)=$ $\int d Z e^{\frac{i}{\hbar} P Z}\left\langle R-\frac{Z}{2}|\hat{O}| R+\frac{Z}{2}\right\rangle$.
} 
authors' familiarity with it, but also because it allows to describe essentially all the relevant aspects mentioned so far and because it is one of the few cases in which the relationship with alternative schemes (in particular mixed Liouville dynamics) has been explored. We shall begin with reviewing the key steps in the derivation of LAND-Map, present in some detail the algorithm, and then show one illustrative application. Finally, in the Conclusions, we shall summarise a few more open questions in this field.

\section{Linearised Non Adiabatic Dynamics in the Mapping Representation (LAND-Map)}

LAND-Map $[19,20]$ is a method targeted at computing approximate time correlation functions. Its starting point is the standard form of the quantum thermal time correlation function of operators $\hat{A}$ and $\hat{B}$

$$
\langle\hat{A}(0) \hat{B}(t)\rangle=\operatorname{Tr}\left[\hat{\rho} \hat{A} e^{\frac{i}{\hbar} \hat{H} t} \hat{B} e^{-\frac{i}{\hbar} \hat{H} t}\right]
$$

where $\operatorname{Tr}$ is the trace operation and $\hat{\rho}=e^{-\beta \hat{H}} / Z$ (with $Z=\operatorname{Tr} e^{-\beta \hat{H}}$ ) is the quantum thermal density. We begin by representing the correlation function in a basis defined as the tensor product $\left|R_{0} \alpha^{\prime}\right\rangle$, where $\tilde{R}_{0}$ are the nuclear coordinates and $\alpha^{\prime}$ indicates the electronic basis which, in contrast to Surface Hopping, has been chosen independent on the nuclear coordinates (this is the so-called diabatic representation of the electronic states). To simplify the discussion, in the following we shall consider the case of operators that are diagonal in the nuclear coordinates. Inserting resolutions of the identity, e.g. $\mathbf{1}=\sum_{\alpha^{\prime}} \int d \tilde{R}_{0}\left|\tilde{R}_{0} \alpha^{\prime}\right\rangle\left\langle\tilde{R}_{0} \alpha^{\prime}\right|$, the expression above can be identically rewritten as

$$
\begin{aligned}
\langle\hat{A}(0) \hat{B}(t)\rangle= & \sum_{\alpha, \beta, \alpha^{\prime}, \beta^{\prime}} \int d R_{0} d R_{N} d \tilde{R}_{0}\left\{\left\langle R_{0} \alpha|\hat{\rho} \hat{A}| \tilde{R}_{0} \alpha^{\prime}\right\rangle\left\langle\tilde{R}_{0} \alpha^{\prime}\left|e^{\frac{i}{\hbar} \hat{H} t}\right| R_{N} \beta^{\prime}\right\rangle\right. \\
& \left.\times B_{\beta^{\prime} \beta}\left(R_{N}\right)\left\langle R_{N} \beta\left|e^{-\frac{i}{\hbar} \hat{H} t}\right| R_{0} \alpha\right\rangle\right\}
\end{aligned}
$$

where $B_{\beta^{\prime} \beta}\left(R_{N}\right)=\left\langle\beta^{\prime}\left|\hat{B}\left(R_{N}\right)\right| \beta\right\rangle\left(\hat{B}\left(R_{N}\right)\right.$ is still an operator in the electronic set). Equation (2) can be read as a sequence of propagations (evaluations of matrix elements of exponentials of the Hamiltonian) and evaluations of the operators' matrix elements. Starting from the right end of the integrand, the prescription is to select a state with electronic state equal to $\alpha$ and a nuclear configuration $R_{0}$ and evaluate the probability amplitude to find it in state $\beta$ and at configuration $R_{N}$ after a time evolution of length $t$. Note that, since we are considering non-adiabatic systems, state $\beta$ needs not be equal to $\alpha$. The matrix element of $\hat{B}$ is then computed. Due to the non diagonal nature of the operator in the electronic states, this operation can change the state label of the system to $\beta^{\prime}$. The state $\left|R_{N} \beta^{\prime}\right\rangle$ is then evolved backward in time for a time $t$ and the probability amplitude to be in state $\left|\tilde{R}_{0} \alpha^{\prime}\right\rangle$ is computed. The trace operation is closed by evaluating the matrix element of the product $\hat{\rho} \hat{A}$ between the final state of the backward propagation and the state, $\left|R_{0} \alpha\right\rangle$, selected as the starting point for the evaluation of the integrand. This set of operations must then be repeated for all possible choices of the electronic states $\alpha, \beta, \alpha^{\prime}, \beta^{\prime}$ and nuclear configurations $R_{0}, R_{N}, \tilde{R}_{0}$ to implement the sums and the integrals in Eq. (2).

The exact numerical evaluation of the expression above is impossible: the evaluation of the propagators is fully equivalent to the solution of the time dependent 
Schrödinger equation for the system and therefore scales exponentially with the number of degrees of freedom. To make progress, LAND-Map uses three steps. The first two are exact and their goal is to express the propagators in a form more suitable for approximations. The third step contains the key approximation of the method and leads to a computable expression for the time correlation function. These three steps are outlined below to summarise the basic ideas and the formal tools used in LANDMap. A detailed derivation of the results we present can be found in the literature. See in particular [19] for a complete derivation and the Appendix in [21] or the book chapter by Coker and Bonella in [22] for a more detailed summary.

- Step 1: The mapping formalism. This step focuses on simplifying the electronic part of the problem by introducing a representation more suitable to the mixed quantum classical treatment. The idea [23-27] is to associate to each electronic state a state in a new, auxiliary, space. This space is defined via the tensor product of $\nu+1$ fictitious harmonic oscillators with unit mass and with principal quantum number $n$ restricted to take values 0 and 1 . More in detail

$$
|\alpha\rangle \rightarrow\left|0_{0}, \ldots, 1_{\alpha}, \ldots, 0_{\nu}\right\rangle \equiv\left|m_{\alpha}\right\rangle .
$$

Thus, the auxiliary state associated to electronic state $|\alpha\rangle$ has all oscillators in the ground state, except for the one in position $\alpha$ in the tensor product. This oscillator, and only this one, is in the first excited state. The representation of the electronic Hamiltonian is also changed by first using a dyadic representation

$$
\hat{h}_{e l}(\hat{R}, \hat{r}, \hat{p})=\sum_{\lambda \mu}|\lambda\rangle\left\langle\lambda\left|\hat{h}_{e l}(\hat{R}, \hat{r}, \hat{p})\right| \mu\right\rangle\left\langle\mu\left|\equiv \sum_{\lambda \mu} \hat{h}_{\lambda \mu}(\hat{R})\right| \lambda\right\rangle\langle\mu|
$$

and then mapping each element of the dyad into a product of creation and annihilation operators in the space of the fictitious oscillators:

$$
|\lambda\rangle\langle\mu| \rightarrow \hat{a}_{\lambda}^{\dagger} \hat{a}_{\mu}
$$

where, for example, $\hat{a}_{\mu}=\left(\hat{q}_{\mu}+i \hat{p}_{\mu}\right) / \sqrt{2 \hbar}$. The mapping Hamiltonian is then defined as

$$
\hat{h}_{m}(\hat{R})=\sum_{\lambda \mu} \hat{h}_{\lambda \mu}(\hat{R}) \hat{a}_{\lambda}^{\dagger} \hat{a}_{\mu} .
$$

Note that the $\hat{h}_{\lambda \mu}(\hat{R})$ are still the matrix elements of the electronic Hamiltonian in the original diabatic basis: they depend on the nuclear position and their functional form is completely general. With the prescriptions above, it is possible to define a new nuclear-mapping Hamiltonian as

$$
\hat{H}_{m}=\hat{P}^{2} / 2 M+\hat{h}_{m}(\hat{R}) .
$$

This mapping ensures that, for example

$$
\left\langle R_{N} \beta\left|e^{-\frac{i}{\hbar} \hat{H} t}\right| R_{0} \alpha\right\rangle=\left\langle R_{N} m_{\beta}\left|e^{-\frac{i}{\hbar} \hat{H}_{m} t}\right| R_{0} m_{\alpha}\right\rangle .
$$

This is the main result of the mapping formalism. It states that the time propagators in the exact expression for the time correlation function can be computed, without affecting the result, using the mapping. Furthermore, this mapping has the very convenient property that, if the creation and annihilation operators are expressed in terms of the positions and momenta operators of the fictitious harmonic oscillators, the map of the electronic Hamiltonian is given by a quadratic function of these new operators. 
- Step 2: Path integral representation of the propagators. To move towards a picture of the dynamics based on trajectories, in LAND-Map the nuclear-mapping propagators, e.g. Eq. (8), are expressed using the path integral formalism introduced by Feynman [28-30]. Within this picture, the propagators can be formally written as a sum over all possible sequences of values of the coordinates and momenta of the system that take it from the initial (the ket $\left|R_{0} m_{\alpha}\right\rangle$ ) to the final (the bra $\left.\left\langle R_{N} m_{\beta}\right|\right)$ state in the assigned time $t$. These sequences, known as paths, are identified by first discretising the time interval $t$ in $N$ steps, and then denoting the coordinates and momenta at step $k$ as $R_{k}, P_{k}(k=1, \ldots, N)$. Coordinates and momenta at $k$ and $k+1$ are separated by a time interval $\delta_{t}=t / N$, but there is no specific relation between successive values of these variables ${ }^{4}$. In the following we will indicate one of the (discrete) paths as $\left(\left\{R_{k}\right\},\left\{P_{k}\right\}\right)(k=0, \ldots, N)$. Each path in the sum must be weighted by an appropriate phase factor, given by the classical action along the path. As mentioned above, the mapping Hamiltonian is quadratic in the oscillator's degrees of freedom. This makes it possible to calculate exactly the path integral evolution for the mapping subsystem (the calculation is analogous to that of the propagator for an harmonic system with a time dependent frequency [30]). For each nuclear path, this evolution can be in fact expressed in terms of an integral over a set of classical trajectories for the coordinates and momenta of the mapping degrees of freedom. The nuclear path integral, on the other hand, cannot be computed exactly for general systems. More explicitly, the path integral form of the propagator is

$$
\begin{aligned}
& \left\langle R_{N} m_{\beta}\left|e^{\frac{i}{\hbar} \hat{H}_{m} t}\right| R_{0} m_{\alpha}\right\rangle=\sum_{(\text {paths })} e^{\frac{i}{\hbar} S\left(\left\{R_{k}\right\},\left\{P_{k}\right\}\right)} \\
& \quad \times \int d q_{0} d p_{0} w_{\alpha \beta}\left(q\left(t_{k}\right), p\left(t_{k}\right), q_{0}, p_{0} ;\left\{R_{k}\right\}\right) r_{\alpha}\left(q_{0}, p_{0}\right) G\left(q_{0}, p_{0}\right) \\
& \quad \times e^{-i \theta_{\beta}\left(\left(q\left(t_{k} ;\left\{R_{k}\right\}\right), p\left(t_{k} ;\left\{R_{k}\right\}\right)\right)\right)} .
\end{aligned}
$$

In the expression above, we have written, following conventional notation in this formalism $[29,30]$, the sum over all possible nuclear paths as $\sum_{(\text {paths })}$. This expression becomes exact in the limit $N \rightarrow \infty^{5}$. The notation indicates that for finite $N$ the paths are discrete so, for example, $R_{k}$ indicates the coordinate at time $t_{k}=k \times \delta_{t}$ along the propagation and successive positions and momenta are separated by a time interval $\delta_{t}=t / N$. The mapping propagator, instead, is written as the integral over the initial conditions $q_{0}, p_{0}$ for a set of classical mapping trajectories $\left(q\left(t_{k} ;\left\{R_{k}\right\}\right), p\left(t_{k} ;\left\{R_{k}\right\}\right)\right)$ that depend parametrically on the nuclear path (the Hamiltonian for this classical propagation will be given below). In this writing, $q_{0} \equiv\left\{q_{00}, \ldots, q_{\alpha 0}, \ldots, q_{\nu 0}\right\}$ is the $\nu$-dimensional vector of the initial coordinates of the mapping oscillators with analogous notation for they momenta.

\footnotetext{
4 This is a consequence of the procedure used to construct the path integral representation. Considering for simplicity a representation in the coordinates, $x$, alone, we can use the time composition property of the propagator and resolutions of the identity in the coordinate basis to write, exactly, $\left\langle x_{N}\left|e^{-\frac{i}{\hbar} \hat{H} t}\right| x_{0}\right\rangle=\prod_{k=0}^{N-1} \int d x_{k}\left\langle x_{k+1}\left|e^{-\frac{i}{\hbar} \hat{H} \delta_{t}}\right| x_{k}\right\rangle$. A path is one of the sequences $\left\{x_{k}\right\}_{k=0, \ldots, N}$ that appear in the integrals. Since, for each $x_{k}$, an integral over all possible values of $x_{k+1}$ must be performed, there is no specific relationship among the values of the coordinates at subsequent steps along the path.

${ }^{5}$ It should be mentioned that the mathematical conditions under which the limit $N \rightarrow \infty$ for the path integral representation of the propagator is well defined are not clear and indeed, for general potentials, this may even be an ill defined notion. In this work, as in most of the path integral related work in physics and chemistry, we shall simply assume that this is not the case.
} 
The explicit form of the (real) functions $S\left(\left\{R_{k}\right\},\left\{P_{k}\right\}\right), \theta_{\beta}\left(\left(q\left(t_{k} ;\left\{R_{k}\right\}\right)\right.\right.$, $\left.\left.p\left(t_{k} ;\left\{R_{k}\right\}\right)\right)\right), r_{\alpha}, G\left(q_{0}, p_{0}\right)$, and $w_{\alpha \beta}\left(q\left(t_{k}\right), p\left(t_{k}\right), q_{0}, p_{0} ;\left\{R_{k}\right\},\right)$ is not crucial (see [19] for details), except to say that $w$ is a positive definite function as is $r_{\alpha}$, while $G\left(q_{0}, p_{0}\right)$ is a multidimensional Gaussian function, and to note that Eq. (9) contains two phase factors. The idea to express quantum propagation in terms of paths is intuitively appealing since it reintroduces a phase space picture in quantum mechanics. In practice, however, the summation over the nuclear paths cannot be carried out analytically except for very simple potentials. The path integral expression of the propagator is also extremely difficult, if not impossible, to compute numerically. This is due to two main reasons that reflect the exponential cost of exact solution methods. Firstly, the notion of a path is not easy to transfer to an algorithm. Indeed, there is no prescription for generating the time sequence of coordinates and momenta (in particular, a path is not a classical trajectory so it cannot be obtained via standard molecular dynamics methods). Secondly, the phase factors can cause very rapid oscillations which make convergence exponentially expensive with time and number of degrees of freedom. This pathology is known as the dynamical sign problem.

- Step 3: Linearization approximation. Substituting Eq. (9) and the analogous expression for the backward propagator in Eq. (2) gives an exact expression for the correlation function in which the time evolution is written in terms of sums over forward and backward nuclear paths and classical motion for the mapping variables. LAND-Map approximates this result by assuming that the most important contributions to these sums are given by pairs of forward and backward nuclear paths that remain close during the propagation. Formally, the approximation is enforced by changing variables to the semi-sum and difference nuclear paths. Indicating with $R_{k}$ and $\tilde{R}_{k}$ the coordinates visited at time $t_{k}$ along a forward and backward path, respectively, the semi-sum path is defined as $\bar{R}_{k}=\left(R_{k}+\tilde{R}_{k}\right) / 2$ and the difference path is $\Delta_{k}=R_{k}-\tilde{R}_{k}$, for $k=1, \ldots, N$, with analogous definitions for the momenta. The phase of the correlation function is then expanded to first order in the difference variables. With this expansion, the integrals over the difference paths can be performed analytically resulting in a product of delta functions (see third line in Eq. (10) below). The arguments of the delta functions are such that the only non-zero contributions to the correlation function are given by sequences of coordinates and momenta that satisfy ${ }^{6} R_{k}=R_{k-1}+\delta_{t} P_{k-1} / M$ and $P_{k}=P_{k-1}+\delta_{t} F_{\beta \beta^{\prime}}\left(R_{k-1}\right)$. Thus, once the initial conditions $R_{0}, P_{0}$ are chosen, the sequence of semi-sum variables is uniquely determined by the approximation and can be interpreted as a nuclear trajectory. This trajectory is determined by the approximation and not imposed a priori as, for example, in Surface Hopping. Note that this approximation tackles simultaneously the two main difficulties of path integral calculations: the linearisation of the phase mitigates the oscillations (in the spirit of a stationary phase approximation) of the integrand and the delta functions determine uniquely the semi-sum paths to be computed eliminating the problem of finding an effective way to generate them. Furthermore, the approximation can be interpreted (if not justified) in terms of the physical characteristics of the system. In [21] it was shown that the phase expansion in the difference variables is equivalent to an expansion of the phase in the parameter $\mu=\sqrt{m / M}$, i.e. in the ratio of the masses of the quantum subsystem and the bath.

\footnotetext{
${ }^{6}$ See Eq. (10) and the text following it for a more detailed discussion, and Eq. (12) for the definition of $\left.F_{\beta \beta^{\prime}}\left(R_{k-1}\right)\right)$.
} 


\section{The algorithm}

Implementing the steps outlined in the previous section leads to the following approximation for the time correlation function

$$
\begin{aligned}
\langle\hat{A} \hat{B}(t)\rangle_{L M}= & \sum_{\alpha \beta \alpha^{\prime} \beta^{\prime}} \int d q_{0} d p_{0} d \tilde{q}_{0} d \tilde{p}_{0} \int d R_{0} d P_{0} \int \prod_{k=1}^{N} d R_{k} \frac{d P_{k}}{2 \pi \hbar} B_{\beta \beta^{\prime}}\left(R_{N}\right) \\
& \times w_{\alpha \beta}\left(q\left(t_{N}\right), p\left(t_{N}\right)\right) \tilde{w}_{\alpha^{\prime} \beta^{\prime}}\left(\tilde{q}\left(t_{N}\right), \tilde{p}\left(t_{N}\right)\right) e^{-i \delta_{t} \sum_{k=0}^{N}\left(\theta_{\beta}\left(R_{k}\right)-\tilde{\theta}_{\beta^{\prime}}\left(R_{k}\right)\right)} \\
& \times \prod_{k=1}^{N} \delta\left(\frac{P_{k}-P_{k-1}}{\delta_{t}}-F_{\beta \beta^{\prime}}\left(R_{k-1}\right)\right) \delta\left(\frac{R_{k}-R_{k-1}}{\delta_{t}}-\frac{P_{k-1}}{M}\right) \\
& \times r_{\alpha}\left(q_{0}, p_{0}\right) G\left(q_{0}, p_{0}\right) r_{\alpha^{\prime}}\left(\tilde{q}_{0}, \tilde{p}_{0}\right) G\left(\tilde{q}_{0}, \tilde{p}_{0}\right)[\hat{\rho} \hat{A}]_{\alpha \alpha^{\prime}}^{w}\left(R_{0}, P_{0}\right) .
\end{aligned}
$$

Above, $R_{k}$ is a variable along the semi-sum path defined in the previous section. We have dropped the bar from the symbol to simplify the notation. The structure of this approximation can be reconstructed via the following observations. The sum over the electronic state labels in the first line has remained unchanged with respect to the exact expression in Eq. (2) and depends on the choice of the basis set. The integrals over the mapping variables originate from the path integral expression of the mapping propagator. Within this representation, the paths are classical trajectories governed by an Hamiltonian of the form $[19,21,22]$

$$
h_{m}=\frac{1}{2} \sum_{\lambda} h_{\lambda \lambda}(R)\left(q_{\lambda}^{2}+p_{\lambda}^{2}\right)+\frac{1}{2} \sum_{\lambda \neq \lambda^{\prime}} h_{\lambda \lambda^{\prime}}(R)\left(q_{\lambda} q_{\lambda^{\prime}}+p_{\lambda} p_{\lambda^{\prime}}\right) .
$$

As mentioned in the previous section, the mapping path propagators are exact due to the quadratic nature of the mapping Hamiltonian. Two mapping paths appear above, variables with and without tilde, corresponding to the fact that the correlation function contains two time evolution operators (the forward and backward propagators in Eq. (2)). The double propagation of the electronic degrees of freedom is maintained in the LAND-Map approximation. The integrals over the nuclear coordinates and momenta, on the other hand, reflect the key approximation of the method. The two propagators in the exact correlation function (which would correspond to two infinite sets of paths forward and backward in time) have in fact been reduced to a single path. Furthermore, the values of the coordinates and momenta along this path are uniquely determined, for each choice of $R_{0}, P_{0}$, by the product of delta functions in the third line of Eq. (10). These delta functions enforce a time stepping prescription to identify $R_{k}, P_{k}$ (see step (6) in the description of the algorithm below) reminiscent of classical propagation. The "force" $F_{\beta \beta^{\prime}}(R)$ is however non classical and is given by $[19,22]$

$$
\begin{aligned}
F_{\beta \beta^{\prime}}(R)= & -\frac{1}{2}\left[\nabla_{R} h_{\beta \beta}(R)+\nabla_{R} h_{\beta^{\prime} \beta^{\prime}}(R)\right] \\
& -\frac{1}{2} \sum_{\lambda} \nabla_{R} h_{\beta \lambda}(R)\left[\frac{q_{\beta} q_{\lambda}+p_{\beta} p_{\lambda}}{q_{\beta}^{2}+p_{\beta}^{2}}\right]
\end{aligned}
$$




$$
-\frac{1}{2} \sum_{\lambda^{\prime}} \nabla_{R} h_{\beta^{\prime} \lambda^{\prime}}(R)\left[\frac{\tilde{q}_{\beta^{\prime}} \tilde{q}_{\lambda^{\prime}}+\tilde{p}_{\beta^{\prime}} \tilde{p}_{\lambda^{\prime}}}{\tilde{q}_{\beta^{\prime}}^{2}+\tilde{p}_{\beta^{\prime}}^{2}}\right] .
$$

(The dependence on the forward and backward variables has been omitted from the argument of $F$ for notational convenience.) The structure of the equation above, which is entirely determined by the linearisation, has several interesting features. The "force" experienced by the nuclei is different for different choices of the electronic state labels $\beta$ and $\beta^{\prime}$, i.e. it depends on the specific term of the sum in Eqs. (2) and (10) via the final states in the forward and backward propagation. If $\beta=\beta^{\prime}$ the first line in the equation above indicates that the nuclei move on a single state (i.e. diagonal element of the electronic Hamiltonian) in a way reminiscent of Surface Hopping, but if $\beta \neq \beta^{\prime}$ the evolution occurs on a mean surface, in a way reminiscent of Ehrenfest dynamics. This pure state or mean propagation is, however, dressed by the terms in the second and third line above which, in principle, involve all the electronic states. These terms are non zero when the off-diagonal elements of the electronic Hamiltonian are non zero and not constant with respect to changes in the nuclear coordinate. This corresponds to regions of non zero couplings among the electronic states. Thus, these terms in the force represent the feedback on the nuclear motion of the amplitude transfer among electronic states which occurs when non adiabatic transitions take place. This transfer, in turn, is obtained by evolving the mapping variables. In fact, it can be shown $[19,21,22]$ that the population of state $\alpha$ (i.e. the probability to find the electronic system in state $\alpha$ ) in the mapping representation is given by $q_{\alpha}^{2}+p_{\alpha}^{2}$. Given the hamiltonian in Eq. (11), this quantity is constant outside of the coupling regions (i.e. for $h_{\beta \lambda}(R)=0$ ) and changes when the coupling is non zero. This concludes the description of the coupled evolution in LAND-Map, which is the main ingredient of the method. The other terms in Eq. (10) can be described as follows. The product of functions in the second line of the expression can be interpreted as a weight to be associated with each trajectory, while the product of functions in the last line provides us with a probability density to sample initial conditions for the mapping variables, from $r_{\alpha}\left(q_{0}, p_{0}\right) G\left(q_{0}, p_{0}\right) r_{\alpha^{\prime}}\left(\tilde{q}_{0}, \tilde{p}_{0}\right) G\left(\tilde{q}_{0}, \tilde{p}_{0}\right)$, a product of positive definite functions that allow for direct sampling, and the nuclear phase space, from the absolute value of $[\hat{\rho} \hat{A}]_{\alpha \alpha^{\prime}}^{w}\left(R_{0}, P_{0}\right)$, the Wigner transform of the product of the thermal density times the operator $\hat{A}$. This last sampling is non trivial. The Wigner transform is in fact difficult to calculate for generic densities and observables. Several approximate methods have, however, been developed for this purpose using either harmonic approximations of the thermal density whose Wigner transform is known analytically (see next subsection for a typical example), or keeping the exact form of the density but implementing quite effective numerical approximations [31-34] for the Wigner sampling.

From the observations above, Eq. (10) can be calculated via the following algorithm, which combines Monte Carlo sampling of the initial conditions with classical like evolution.

1. Assign a set of indices $\alpha, \alpha^{\prime}, \beta, \beta^{\prime}$.

2. Sample initial values of the nuclear positions and momenta $\left(R_{0}, P_{0}\right)$ from the Wigner transform of the thermal density times the operator $\hat{A}$.

3. Sample initial values of the forward and backward mapping variables $\left(q_{0}, p_{0}\right)$ and $\left(\tilde{q}_{0}, \tilde{p}_{0}\right)$ from $G$ and $\tilde{G}$.

4. Accumulate weights, i.e. $e^{i\left(\theta_{\alpha}-\theta_{\alpha^{\prime}}\right)}$ at initial time. 
5. Advance mapping variables via

$$
\begin{aligned}
& q_{\beta 1}=q_{\beta 0}+\delta_{t}\left[h_{\beta \beta}\left(R_{0}\right) p_{\beta 0}+\sum_{\lambda \neq \beta} h_{\beta \lambda}\left(R_{0}\right) p_{\lambda 0}\right] \\
& p_{\beta 1}=p_{\beta 0}-\delta_{t}\left[h_{\beta \beta}\left(R_{0}\right) q_{\beta 0}+\sum_{\lambda \neq \beta} h_{\beta \lambda}\left(R_{0}\right) q_{\lambda 0}\right]
\end{aligned}
$$

with an analogous prescription for the tilde variables.

6. Advance nuclear positions and momenta according to the argument of the delta functions using

$$
\begin{aligned}
& R_{1}=R_{0}+\delta_{t} \frac{P_{0}}{M} \\
& P_{1}=P_{0}+\delta_{t} F_{\beta \beta^{\prime}}\left(R_{0}, q_{\beta 0}, p_{\beta 0}, \tilde{q}_{\beta 0}, \tilde{p}_{\beta 0}\right) .
\end{aligned}
$$

7. Compute the matrix elements $h_{\beta \lambda}$ at the new nuclear position.

8. Accumulate weights, in particular $e^{i \delta_{t}\left(\theta_{\beta}\left(R_{1}\right)-\theta_{\beta^{\prime}}\left(R_{1}\right)\right)}$, and iterate from step (5) until the final time of the run is reached (i.e. until $\mathrm{N}$ time steps have been performed).

9. Compute weights $w_{\alpha \beta}$ and $\tilde{w}_{\alpha^{\prime} \beta^{\prime}}$ and observable $B_{\beta \beta^{\prime}}$ at the end points of the propagation.

10. Iterate from step (1) until the result does not change (within error bars) by increasing the number of trajectories.

Note that the weight accumulated in step (8) is a phase factor. Its presence is key to represent, within the limits of the linearisation approximation, interference effects among different trajectories. These interferences approximate, for example, electronic quantum coherence and represent a distinct improvement with respect to ad hoc methods for which coherence is either absent or reintroduced "by hand" in the calculation. On the other hand, the phase factor can, and in most cases does, reintroduce oscillations in the expression of the correlation function. The need to accurately capture the effect of these oscillations, that grow faster with time and dimensions of the system, imposes very large number of trajectories (of the order of tens to hundreds of thousand depending on the size of the system) to converge. Due to this, for example coupling of LAND-Map with ab initio electronic structure methods is basically impossible and current calculations are essentially restricted to systems for which an empirical parametrisation of the electronic Hamiltonian exists. The development of methods to control the phase oscillations via filtering schemes $[35,36]$ or alternative methods such as cumulants expansions $[37,38]$ to pre average the phase, is an active field of research. It remains to be seen if these developments will be sufficient to make the numerical cost of this method comparable to that of ad hoc schemes.

\subsection{Illustrative application: The Spin Boson model}

A very important benchmark system for formally derived mixed methods and in particular for LAND-Map is the spin boson problem. This model describes the dissipative dynamics of a two level system (the quantum subsystem in the mixed quantum classical scheme) coupled to an environment (the bath). In calculations on the spin boson model, the environment is represented by a discrete set of $N_{o}$ harmonic oscillators, linearly coupled to the quantum degree of freedom. In particular, we will show results for $N_{o} \in\{10,11, \ldots, 20\}$. The characteristics of the bath are specified by the spectral 

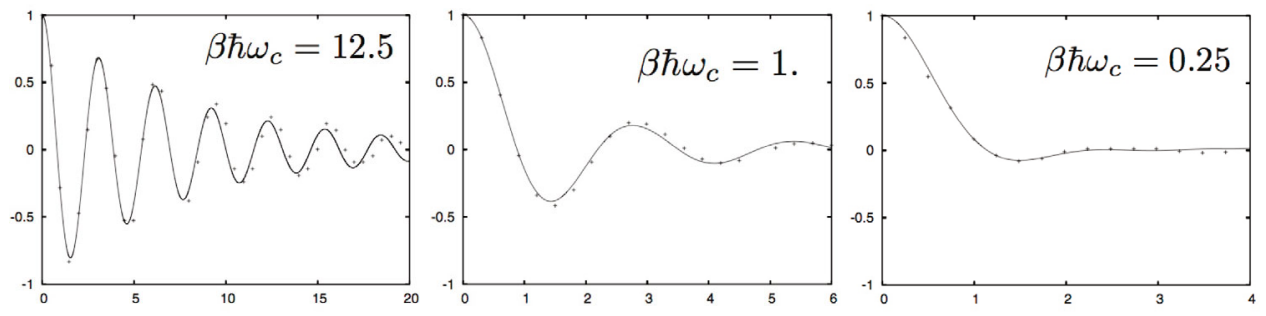

Fig. 1. $D(t)$ as a function of $\Omega t$ in the weak coupling regime. The solid curve is the LANDMap result, while the points were obtained with numerically exact calculations $[42,44,46]$. In all figures, $\Omega / \omega_{c}=0.4$ and $\xi \approx 0.1$. The different temperatures (expressed in the relevant energy scale) of the calculations are indicated in the insets, with the lowest temperature on the left.

density, which we will assume of Ohmic form $J(\omega)=\xi \omega e^{-\omega / \omega_{c}}$, where $\xi$ is the Kondo parameter (specifying the strength of the coupling) and $\omega_{c}$ is the frequency at which the spectral density has a maximum. With an appropriate choice of units $[16,39]$, the Hamiltonian of the system is

$$
\hat{H}=-\Omega \hat{\sigma}_{x}+\sum_{j=1}^{N_{o}}\left[\frac{P_{j}^{2}}{2}+\frac{1}{2} \frac{\omega_{j}^{2}}{\omega_{c}^{2}} R_{j}^{2}-c_{j} R_{j} \hat{\sigma}_{z}\right]
$$

where $\hat{\sigma}_{x, z}$ are Pauli matrices, $\omega_{j}$ is a set of frequencies sampled from $J(\omega)$, $c_{j}=\omega_{j}{\sqrt{\xi \omega_{0}}}^{7}$, and $2 \hbar \Omega$ is the gap between the two levels in the bare quantum system. Initial conditions for this model are sampled from an uncorrelated systembath probability density of the form $\hat{\rho}_{s b}=\rho_{w}\left(R_{0}, P_{0}\right)|1\rangle\langle 1|$, where the Wigner transform for the bath degrees of freedom is known analytically and given by $\rho_{w}\left(R_{0}, P_{0}\right)=\prod_{j=1}^{N_{o}} \frac{\tanh \left(\beta \omega_{j} / 2\right)}{\pi} e^{-\tanh \left(\beta \omega_{j} / 2\right) / 2 \omega_{j}\left[P_{0 j}^{2}+\omega_{j}^{2} R_{0 j}^{2}\right]}$. The relevant observable is the, time dependent, difference in the populations of the two quantum levels. This quantity is given by $D(t)=\left\langle\hat{\sigma}_{z}(t) \mathbf{1}_{\text {bath }}\right\rangle^{8}$ (the bracket indicates the average on $\hat{\rho}_{s b}$ ). Depending on the temperature and the value of the Kondo parameter, $D(t)$ exhibits a variety of behaviours ranging from coherent oscillations (low temperature, small $\xi$ ) to over damped relaxation (low temperature, large $\xi$ ). Furthermore, reliable numerical calculations [40-46], using the path integral formalism and in particular the influence functional approach, have been performed for this system and can be used as a benchmark. In the following, to exemplify the performance of linearised methods for this kind of system, we summarise results in two interesting regimes. Further details of these calculations can be found in [19]. In Fig. 1, we show results in the weak coupling regime with temperatures varying over three orders of magnitude. Agreement between LAND-Map and exact results is very good at all temperatures. The somewhat surprising agreement even at very low temperatures (left panel in the figure) can be rationalised by observing that, for this system, the linearisation approximation becomes exact in the limit of zero coupling. In this limiting case, in fact, the bath is described by a set of uncoupled harmonic oscillators for which linearisation is exact, and the dynamics of the quantum subsystem is also represented exactly via the mapping formalism. The small value of the coupling is then responsible for the good agreement. The limits of the linearisation approximation are more evident for larger couplings. To exemplify them, in Fig. 2 we show more results for a spin-boson

\footnotetext{
${ }^{7} \omega_{0}$ is a parameter introduced to sample the frequencies from $J(\omega)$, see $[19,41]$.

8 The interpretation of the observable as population difference is immediate remembering that $\hat{\sigma}_{z}=|1\rangle\langle 1|-| 2\rangle\langle 2|, \mathbf{1}_{\text {bath }}$ is the identity in the bath space.
} 

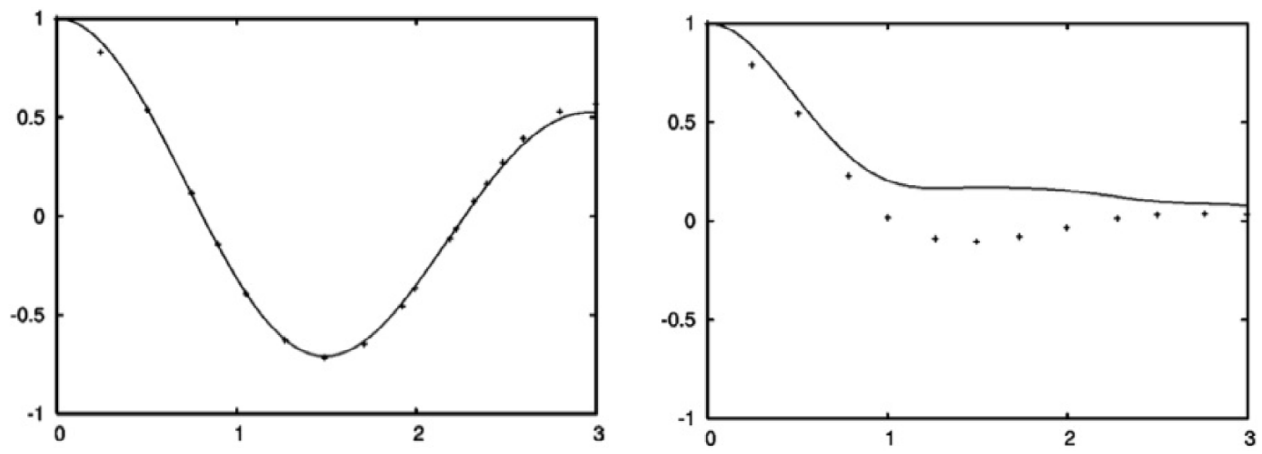

Fig. 2. $D(t)$ as a function of $\Omega t$ in the strong coupling regime at fixed $\beta \hbar \omega_{c}=3$ (low temperature). In the left panel, $\xi=0.1$. In the right panel, $\xi=0.5$. The solid curve is the LAND-Map result, while the points were obtained with numerically exact calculations [45]. In all figures, $\Omega / \omega_{c}=1 / 3$.

system at low temperature. In the left panel, the value of the Kondo parameter is small and, as before, our method reproduces the exact result. In the right panel, however, we see a large discrepancy between the results of the linearised calculation and exact values. In this case, the friction is relatively large, but our linearisation approximation overemphasises its effect and underestimates the importance of the coherent dynamics. Thus the exact results oscillate around zero while the linearised approximate results show slow incoherent decay.

The numerical effort required by LAND-Map calculations for this system is very small: the calculations reported reproduced the numerically exact result with about 500 trajectories.

The results reported in this section, which were chosen because they still represent an interesting reference point, can be - and in some cases have been - improved. In particular, in [47], it was shown how to improve agreement at low temperatures and strong coupling by a generalisation of LAND-Map in which the overall dynamics is obtained by concatenating a sequence of linearised propagators of the form shown in the previous section. While this is an interesting development, which results in a systematic procedure to increase the accuracy of the method, it has the important drawback of requiring considerably larger numbers of trajectories (of the order of a million) to converge. More recently, Huo and Coker have proposed a refined version of the method which further improves accuracy of the linearisation and has been successfully applied to model coherent excitation energy transfer in photosynthetic light harvesting [48], and condensed phase electron transfer [49].

\section{Discussion}

This paper contains a brief outline of mixed quantum classical methods. These methods can be broadly divided in two categories: ad hoc schemes and formally derived methods. Two of the most popular ad hoc schemes in the field, Surface Hopping and Ehrenfest dynamics, have been recalled in the Introduction together with a representative of the formally derived approaches, the mixed Liouville method. Another formally derived approach, LAND-Map, has then been described in some detail to illustrate typical problems of exact methods and typical steps to obtain approximate, computable expressions. The LAND-Map algorithm has then been described in some 
detail, indicating both its advantages (the approximate time evolution is reduced to a mixed scheme that combines Monte Carlo and classical like propagation) and its difficulties (the estimator of the correlation function contains phase factors that complicate convergence).

To conclude, it may be interesting to summarise some very general questions and interesting lines for current and future research (the list below is non exhaustive but it contains questions that are more directly related to the contents of this paper):

1. Is it possible to bridge the gap between numerically efficient but poorly justified ad hoc methods and more rigorous but computationally expensive methods such as mixed Liouville or LAND-Map? As mentioned in the Introduction, several groups are at work in this field, in particular attempting to derive Surface Hopping from "first principles", but no definite answer has been put forward yet. If positive, this answer would represent however a major advancement (the field's "Holy Grail"?) by opening the way to combine the best of the worlds of ad hoc and formal methods.

2. Is it possible to derive systematic tools to analyse the range of accuracy of mixed methods? Interestingly, this question affects both families of methods and, in particular, also LAND-Map. In fact, even though the expansion in the mass ratio provides a justification for the linearisation, more work is necessary to assess the time spans that can be tackled via this approximation. (A similar difficulty arises for mixed Liouville dynamics.) This is usually not considered a stumbling block for applications based on the argument that typical calculations focus on quantities (e.g. correlation functions) that decay very rapidly for condensed phase systems and thus only require to capture accurately the short time evolution of the system. While this argument is supported by the success of mixed methods, it would be desirable (and useful) to make a more precise statement.

3. Is it possible to analyse systematically the relationship between the available approaches? One of the characteristics of the field is that a considerable number of methods have been proposed in the last forty years, but very little efforts have been devoted to a systematic comparison among them. This is due both to the fact that the formal starting points of the various approaches are, or appear, very different and to the difficulty, in particular for the ad hoc schemes, of comparing algorithms that do not rely on a precise formal development. Indeed, one of the few cases in which such an analysis has been performed is precisely that of LANDMap and mixed Liouville dynamics. In [21] it was in fact shown that not only the approximation in these approaches is governed by the same physical parameter $(\mu=\sqrt{m / M})$ but that the mixed evolution equations for both schemes could be derived from the same linearisation procedure applied to the exact quantum propagator. This result was useful, for example, to explain the essentially identical numerical performance of the methods on benchmark systems [22]. It also explained some striking similarities in the structure of the generalised forces used to propagate the bath's motion in these approaches. It would be interesting to explore further connection with other approximations schemes. In particular, the $\mu$ expansion of the phase in LAND-Map is reminiscent of a (partial, since it involves only the nuclear degrees of freedom) WKB (see, for example, [51]) approximation of the propagator, but this connection has not been explored so far. In general, setting aside LAND-Map, a more extensive comparison among methods might help to identify the more promising approaches and simplify the field.

To conclude, it is important to mention that all mixed quantum classical methods assume that a purely classical description is sufficient for the dynamics of the bath. This means, in the case of non adiabatic dynamics, that nuclear quantum effects are, by construction, not included. While this is often a reasonable assumption, there are 
physically significant situations (most notably systems that contain hydrogen atoms) for which this hypothesis is not justified. While some attempts to include such effects are being developed for Born-Oppenheimer dynamics [50] using an approach similar to that presented here for LAND-Map, their efficiency is still unsatisfactory and more work is necessary also to understand their formal aspects.

The contents of this paper draw heavily on previous work and material and on discussions with several colleagues. We would like to thank, in particular, I. Tavernelli for input on Surface Hopping. We also thank D. Coker for collaborations on LAND-Map, and R. Kapral for collaborations on Wigner-Liouville dynamics and the mapping formalism.

\section{References}

1. P. Ehrenfest, Zeitung Physik 45, 455 (1927)

2. X. Li, J.C. Tully, H.B. Schlagel, M.J. Frisch, J. Chem. Phys. 123, 084106 (2005)

3. J.C. Tully, J. Chem. Phys. 55, 562 (1971)

4. J.C. Tully, J. Chem. Phys. 93, 1061 (1990)

5. M. Barbatti, Wiley Interdisciplinary Reviews: Computational Molecular Science 1, 620 (2011)

6. E. Tapavicza, I. Tavernelli, U. Rothlisberger, Phys. Rev. Lett. 98, 023001 (2007)

7. I. Tavernelli, E. Tapavicza, U. Rothlisberger, J. Chem. Phys. 130, 124107 (2009)

8. I. Tavernelli, B.F.E. Curchod, U. Rothlisberger, Chem. Phys. 391, 101 (2011)

9. R. Kapral, J. Phys.: Condens. Mat. 27, 1 (2015)

10. F. de Carvalho, M. Bouduban, B. Curchod, I. Tavernelli, Entropy 16, 62 (2014)

11. B.J. Schwartz, E.R. Bittner, O.V. Prezhdo, P.J. Rossky, J. Chem. Phys. 104, 5942 (1996)

12. G. Granucci, M. Persico, A. Zoccante, J. Chem. Phys. 133(13), 134111

13. J. Subotnik, W. Ouyang, B. Landry, J. Chem. Phys. 139, 214107 (2013)

14. R. Kapral, G. Ciccotti, J. Chem. Phys. 110, 8919 (1999)

15. E. Wigner, Phys. Rev. 40, 749 (1932)

16. D.M. Kernan, G. Ciccotti, R. Kapral, J. Phys. Chem. B 112, 424 (2008)

17. H. Kim, A. Nassimi, R. Kapral, J. Chem. Phys. 129, 084102 (2008)

18. S. Nielsen, R. Kapral, G. Ciccotti, J. Chem. Phys. 115, 5805 (2001)

19. S. Bonella, D.F. Coker, J. Chem. Phys. 122, 194102 (2005)

20. S. Bonella, D. Montemayor, D.F. Coker, Proc. Natl Acad. Sci. USA 102, 6715 (2005)

21. S. Bonella, R. Kapral, G. Ciccotti, Chem. Phys. Lett. 484, 399 (2010)

22. D. Coker, S. Bonella, Computer Simulations in Condensed Matter: From Materials to Chemical Biology, Vol. 1, Lecture Notes in Physics (Springer, 2006), p. 553

23. F. Strocchi, Rev. Mod. Phys. 38, 36 (1966)

24. G. Stock, M. Thoss, Phys. Rev. Lett. 78, 578 (1997)

25. G. Stock, M. Thoss, Phys. Rev. A 59, 64 (1999)

26. W.H. Miller, C.W. McCurdy, J. Chem. Phys. 69, 5163 (1978)

27. C.W. McCurdy, H.D. Meyer, W.H. Miller, J. Chem. Phys. 70, 3177 (1979)

28. R.P. Feynman, Rev. Mod. Phys. 20, 367 (1948)

29. H. Kleinert, Path Integrals in Quantum Mechanics, Statistics, and Polymer Physics, and Financial Markets (Oxford University Press, Oxford, 2004)

30. L.S. Schulman, Techniques and Applications of Path Integration (Dover Books on Physics) (Dover Publications, 2005)

31. Q. Shi, E. Geva, J. Phys. Chem. A 107, 9059 (2003)

32. J.A. Poulsen, G. Nyman, P.J. Rossky, J. Chem. Phys. 119, 12179 (2003)

33. J. Liu, W.H. Miller, J. Chem. Phys. 131, 074113 (2009)

34. J. Beutier, D. Borgis, R. Vuilleumier, S. Bonella, J. Chem. Phys. 141, 084102 (2014)

35. V.S. Filinov, Nucl. Phys. B 271, 717 (1986)

36. N. Makri, W.H. Miller, Chem. Phys. Lett. 139, 10 (1987) 
37. M.S. Causo, G. Ciccotti, S. Bonella, R. Vuilleumier, J. Phys. Chem. B 110, 3638 (2006)

38. M. Monteferrante, S. Bonella, G. Ciccotti, Molec. Phys. 109, 3015 (2011)

39. D. Mac Kernan, G. Ciccotti, R. Kapral, J. Chem. Phys. 116, 2346 (2002)

40. C.H. Mak, D. Chandler, Phys. Rev. A 44, 2352 (1991)

41. M. Topaler, N. Makri, J. Chem. Phys. 101, 7500 (1994)

42. R. Egger, C.H. Mak, Phys. Rev. B 50, 15210 (1994)

43. K. Thompson, N. Makri, Chem. Phys. Lett. 291, 101 (1998)

44. X. Sun, H.B. Wang, W.H. Miller, J. Chem. Phys. 109, 7064 (1998)

45. K. Thompson, N. Makri, J. Chem. Phys. 110, 1343 (1999)

46. A. Golosov, D.R. Reichman, J. Chem. Phys. 114, 1065 (2001)

47. E.R. Dunkel, S. Bonella, D.F. Coker, J. Chem. Phys. 129, 114106 (2008)

48. P. Huo, D.F. Coker, J. Chem. Phys. 133, 184108 (2010)

49. P. Huo, T.F. Miller, D.F. Coker, J. Chem. Phys. 139, 151103 (2013)

50. S. Bonella, M. Monteferrante, C. Pierleoni, G. Ciccotti, J. Chem. Phys. 133, 164105 (2010)

51. J.J. Sakurai, Modern Quantum Mechanics (Addison-Wesley, 1994) 